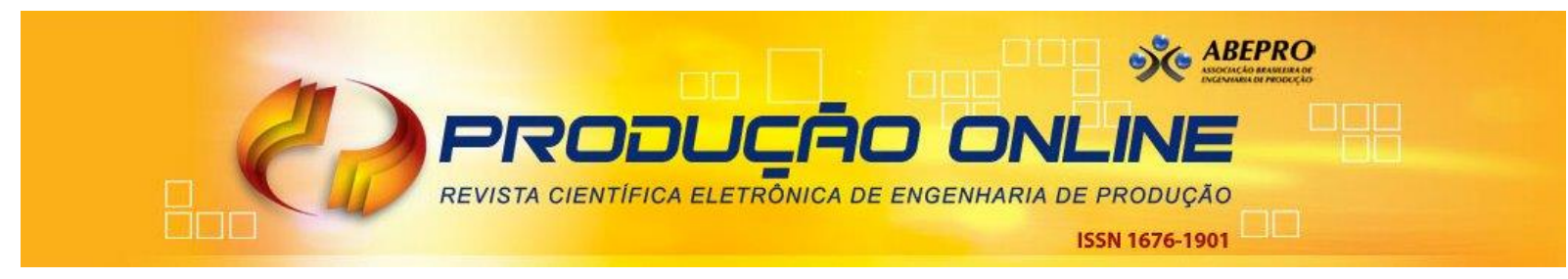

\title{
SEQUENCIAMENTO DA PRODUÇÃO: UMA PROPOSTA PARA O SISTEMA MAKE TO ORDER
}

\section{SCHEDULING: A PROPOSAL FOR MAKE TO ORDER SYSTEM}

\author{
Vanessa Ribeiro * E-mail: vanessar.ibr@gmail.com \\ Nelly H. Marques Cordeiro*E-mail: nelly.marques@grupointergado.br \\ ${ }^{*}$ Centro Universitário Integrado, Campo Mourão, PR
}

\begin{abstract}
Resumo: Um dos objetivos do sequenciamento da produção é a movimentação sincronizada de insumos dentro de um processo produtivo de maneira rápida e ordenada nos recursos disponíveis, visando o processamento de pedidos para atender a demanda do mercado. Diante disso, a presente pesquisa pretende apresentar uma proposta de sequenciamento dos subprodutos que compõe o produto produzido por uma indústria de equipamentos médicos, ao passo que seu sistema de manufatura é classificado como job shop com capacidade finita em um ambiente make to order. A proposta será desenvolvida por meio da análise e caracterização dos processos produtivos; levantamento das restrições; identificação das prioridades da organização, realização da cronoanálise dos subprodutos; e apresentação dos resultados obtidos por uma regra de sequenciamento utilizando o método gráfico de Gantt. Em relação aos procedimentos metodológicos de pesquisa, a abordagem utilizada foi a combinada de triangulação, quanto à sua finalidade é caracterizada como pesquisa aplicada, em relação aos seus objetivos é considerada descritiva, segundo os procedimentos utilizados é classificada como bibliográfica e pesquisa-ação. O resultado obtido foi o sequenciamento dos subprodutos por meio da regra menor tempo de processamento (MTP). As limitações da pesquisa estão relacionadas a escassez de um histórico de informações sobre os resultados da empresa.
\end{abstract}

Palavras-chave: Sequenciamento da produção. Job shop. Make to order. Sistemas com capacidade finita. Gráfico de Gantt.

\begin{abstract}
One of the objectives of scheduling is the synchronized movement of inputs within a production process in a fast and orderly manner in the available resources, aiming at processing orders to meet market demand. Given this, the present research intends to present a proposal for the sequencing of the byproducts that make up the product produced by a medical equipment industry, while its manufacturing system is classified as a job shop with finite capacity in a make to order environment. The proposal will be developed through the analysis and characterization of the productive processes; lifting of restrictions; identification of the priorities of the organization, accomplishment of the chronanalysis of the byproducts; and presenting the results obtained by a sequencing rule using the Gantt graph method. Regarding the methodological research procedures, the approach used was the combined triangulation, as its purpose is characterized as applied research, in relation to its objectives is considered descriptive, according to the procedures used is classified as bibliographic and action research. The result obtained was the sequencing of the byproducts through the rule of shorter processing time (MTP). Research limitations are related to the scarcity of a history of information about company results.
\end{abstract}

Keywords: Scheduling. Job shop. Make to order. Finite capacity systems. Gantt chart. 


\section{INTRODUÇÃO}

É possível constatar que na atualidade as empresas de manufatura enfrentam uma realidade desafiadora, uma vez que os ciclos de vida dos produtos ficam mais curtos com o tempo e as demandas dos clientes mudam constantemente. Sendo assim, ser um produtor de baixo custo não garante mais sucesso como era outrora, pois se faz necessário ofertar produtos de alta qualidade e entregas confiáveis. Nesse contexto, estabelecer uma sequência das operações e conhecer a disponibilidade dos recursos na produção se transformou em preocupações relevantes no âmbito do Planejamento e Controle da Produção (PCP). Diante disso, o sequenciamento da produção (scheduling), umas das atividades do PCP, tem recebido mais atenção de profissionais em ambientes industriais com o propósito de aumentar a competitividade da organização e garantir sua sobrevivência (NOVAS, 2019).

Para Pinedo (2008) scheduling é um processo decisório utilizado em indústrias de manufatura e serviços. Ele trata da alocação de recursos para tarefas que serão executadas em centros de trabalho tendo em vista a produção de saídas nos momentos desejados, satisfazendo assim diversos objetivos simultaneamente. Segundo esse autor, o sequenciamento da produção desempenha um papel fundamental nos sistemas de produção, nos ambientes de processamento de informações e nas configurações de transporte e distribuição. A redução de custos, do tempo de processamento de pedidos (lead time), do tempo de setup, da ociosidade dos equipamentos e colaboradores, dos atrasos nas entregas, maximização dos lucros, otimização dos processos e ganho financeiro são alguns dos benefícios do scheduling.

Durante o processo de sequenciamento é fundamental analisar e caracterizar alguns aspectos da organização, por exemplo: o tipo de capacidade empregada. Segundo Gaither e Frazier (2002) existem duas abordagens a respeito de como as tarefas são atribuídas aos centros de trabalho: a abordagem de capacidade infinita, que é usada quando as capacidades dos centros de trabalho não são consideradas 
durante a atribuição de tarefas; e a abordagem capacidade finita, onde as capacidades dos centros de trabalho são alocadas dentre uma lista de tarefas.

Sobre as prioridades da organização, Slack, Chambers e Johnston (2009)

dizem que um conjunto de regras de sequenciamento pode ser seguido para definir as prioridades dadas ao trabalho em uma operação. Alguns critérios de avaliação são utilizados para saber qual regra tem o melhor desempenho para um grupo de tarefas à espera. Desse modo, é importante realizar uma análise sobre o sistema de produção, ambiente de produção e objetivos de uma organização antes de definir o sequenciamento de suas atividades.

Nesse contexto, um problema de scheduling que vem sendo estudado envolve o sistema de produção job shop, caracterizado por apresentar baixos volumes de produção e elevada variedade de produtos; e a estratégia make to order (MTO), na qual a produção é sob encomenda e geralmente os processos de negócios seguem a seguinte ordem: vender, planejar, produzir e entregar (PIRES, 2004). Nesse cenário há diversos tamanhos de lotes com datas de entregas diferentes para produtos iguais ou similares, além de alterações no projeto de um mesmo item visando atender aos requisitos específicos dos clientes (CKUNGHSING, 2000). Existem alguns métodos utilizados para solucionar o desafio abordado, um deles é o gráfico de Gantt, que conforme Slack, Chambers e Johnston (2009) é um dos métodos mais utilizados para sequenciar tarefas. As vantagens desses gráficos é que eles proporcionam uma visualização rápida do sequenciamento da produção, do que deveria e do que está realmente ocorrendo na operação, informando os tempos de início das atividades e sua duração. Além disso, podem ser utilizados para "testar" sequenciamentos alternativos.

Diante do exposto, a presente pesquisa pretende apresentar uma proposta de sequenciamento dos subprodutos que compõe a autoclave de $100 \mathrm{~L}$ com uma porta produzida pela Cristófoli e Camacho Equipamentos Hospitalares Ltda, localizada no município de Campo Mourão/PR, ao passo que seu sistema de manufatura é classificado como job shop com capacidade finita em um ambiente make to order. A proposta será desenvolvida por meio da análise e caracterização dos processos produtivos; levantamento das restrições; identificação das prioridades da 
organização, realização da cronoanálise dos subprodutos; e apresentação dos resultados obtidos por uma regra de sequenciamento utilizando o método gráfico de Gantt.

A proposta de sequenciamento justifica-se quanto à melhora do desenvolvimento das atividades da produção, tornando o fluxo produtivo mais eficiente e eficaz, aumentando a produtividade, reduzindo custos com recursos humanos e financeiros, da mesma maneira que reduz desperdícios com excesso de movimentação, transporte, espera e processamento inapropriado. A principal contribuição está em apresentar um processo de definição de sequenciamento da produção que possa ser utilizado como referência por empresas de pequeno porte, que ainda não possuem um departamento de PCP estruturado e/ou softwares capazes de sequenciar as tarefas considerando restrições de precedências de uma maneira ótima.

Em relação aos procedimentos metodológicos de pesquisa, a abordagem utilizada foi a combinada de triangulação, quanto à sua finalidade é caracterizada como pesquisa aplicada, em relação aos seus objetivos é considerada descritiva, segundo os procedimentos utilizados é classificada como bibliográfica e pesquisaação.

A presente pesquisa está organizada da seguinte maneira: a seção 2 apresenta uma fundamentação teórica sobre sequenciamento da produção, regras de sequenciamento, sequenciamento de sistemas com capacidade finita e modelos de sequenciamento da produção. A seção 3 é dedicada a apresentação dos procedimentos metodológicos da pesquisa, enquanto que a seção 4 aborda a análise dos dados coletados e os resultados do estudo. As considerações finais da pesquisa são explanadas na seção 5 .

\section{SEQUENCIAMENTO DA PRODUÇÃO}

De acordo com Goldratt e Fox (1989) o sequenciamento da produção pode ser empregado em empresas de manufatura e serviços e está relacionado a outras áreas do PCP, como é o caso da área supply chain. Um dos seus objetivos é a movimentação sincronizada de insumos dentro de um processo produtivo de maneira rápida e ordenada nos recursos disponíveis, visando o processamento de 
pedidos para atender a demanda do mercado. A sequência em que as ordens serão processadas é determinada através das regras de sequenciamento.

\subsection{Regras de Sequenciamento}

Para tratar do problema de sequenciamento é importante compreender o con-

ceito de regra estática e regra dinâmica. Para Moreira (2011) uma regra é considerada estática se determinar a sequência de processamento de todos os trabalhos de uma só vez. Em contrapartida, uma regra é dita dinâmica quando sequencia apenas um trabalho por vez.

Russomano (2000) afirma que definir a prioridade entre os trabalhos é uma tarefa difícil. Sendo assim, durante o processo decisório alguns critérios são utilizados, como: atendimento das datas de entrega; maximização dos lucros; e minimização dos custos. Esses critérios nem sempre são compatíveis, por exemplo, optar pelo critério de atendimento a datas de entrega pode elevar os custos de produção bem acima do nível que se conseguiria se alguns produtos pudessem ser atrasados. A escolha por uma regra também dependerá dos objetivos da organização, do tipo de sistema de produção empregado, além da caracterização da capacidade produtiva e da estratégia de negócio estabelecida.

Para Moreira (2011) os principais critérios de sequenciamento derivam de algumas grandezas. As definições dessas grandezas são apresentadas no Quadro 1.

Quadro 1 - Grandezas analisadas durante o processo de escolha por um critério.

\begin{tabular}{|l|l|}
\hline \multicolumn{1}{|c|}{ ESPECIFICAÇÃo } & \multicolumn{1}{c|}{ DEFINIÇÃo } \\
\hline Tempo de processamento do trabalho (TP) & $\begin{array}{l}\text { É o tempo gasto desde que o trabalho começa a ser } \\
\text { processado até que termina }\end{array}$ \\
\hline Tempo de espera do trabalho (TE) & $\begin{array}{l}\text { É o tempo total que o trabalho espera para que } \\
\text { comece o seu processamento }\end{array}$ \\
\hline Tempo de término do trabalho (TT) & É a soma do TP com o TE \\
\hline Data devida de um trabalho (DD) & É a data na qual o trabalho deveria estar pronto \\
\hline Atraso de um trabalho (AT) & $\begin{array}{l}\text { É a diferença entre TT e DD, desde que TT seja } \\
\text { maior que DD, caso contrário vale zero }\end{array}$ \\
\hline
\end{tabular}

Fonte: Moreira (2011). 
Segundo Slack, Chambers e Johnston (2009) as regras de sequenciamento estabelecem uma lógica para identificar a prioridade de processamento dos lotes de produção em um recurso. Para Tubino (2009) as regras de sequenciamento mais empregadas na prática estão apresentadas na Quadro 2.

Quadro 2 - Regras de sequenciamento em processo em lotes.

\begin{tabular}{|c|c|c|}
\hline SIGLA & ESPECIFICAÇÃO & DEFINIÇÃO \\
\hline PEPS & $\begin{array}{l}\text { Primeira que entra } \\
\text { primeira que sai }\end{array}$ & $\begin{array}{l}\text { Os lotes serão processados de acordo com sua chegada no } \\
\text { recurso }\end{array}$ \\
\hline MTP & $\begin{array}{l}\text { Menor tempo de } \\
\text { processamento }\end{array}$ & $\begin{array}{l}\text { Os lotes serão processados de acordo com os menores } \\
\text { tempos de processamento no recurso }\end{array}$ \\
\hline MDE & Menor data de entrega & $\begin{array}{l}\text { Os lotes serão processados de acordo com as menores datas } \\
\text { de entrega }\end{array}$ \\
\hline IPI & Índice de prioridade & $\begin{array}{l}\text { Os lotes serão processados de acordo com o valor da } \\
\text { prioridade atribuída ao cliente ou ao produto }\end{array}$ \\
\hline \multirow[t]{2}{*}{ ICR } & \multirow[t]{2}{*}{ Índice crítico } & $\begin{array}{l}\text { Os lotes serão processados de acordo com o menor valor de: } \\
\qquad \begin{array}{l}\text { (data de entrega - data atual) } \\
\end{array}\end{array}$ \\
\hline & & Tempo de processamento \\
\hline \multirow[t]{2}{*}{ IFO } & \multirow[t]{2}{*}{ Índice de folga } & $\begin{array}{l}\text { Os lotes serão processados de acordo com o menor valor de: } \\
\text { Data de entrega - } \Sigma \text { tempo de processamento restante }\end{array}$ \\
\hline & & Número de operações restantes \\
\hline IFA & Índice de falta & $\begin{array}{l}\text { Os lotes serão processados de acordo com o menor valor de: } \\
\text { Quantidade em estoque / taxa de demanda }\end{array}$ \\
\hline
\end{tabular}

Fonte: Tubino (2009, p. 117).

Conforme Tubino (2009) a regra PEPS no ambiente industrial eleva o tempo de espera médio dos lotes, isto ocorre porque os lotes com tempos longos atrasam a sequência de produção. A regra MTP gera alguns pontos positivos, um deles é o lead time médio baixo, um ponto negativo é que ordens com tempos elevados de processamento são preteridas. Já a regra MDE reduz os atrasos nas entregas, porém pode fazer com que lotes que poderiam ser concluídos rapidamente fiquem aguardando. Da mesma maneira, a regra IPI apresenta as mesmas características da MDE. Normalmente as regras ICR, IFO e IFA são utilizadas em sistemas informatizados de sequenciamento. 


\subsection{Sequenciamento de Sistemas com Capacidade Finita}

Para Slack, Chambers e Johnston (2009), o sequenciamento da produção em um ambiente com capacidade finita adota uma abordagem em que a alocação das tarefas nos centros de trabalho ocorre até um limite estabelecido. Phanden, Jain e Verma (2012) dizem que o sequenciamento nesse ambiente utiliza condições reais do chão de fábrica, resultando em um cronograma que reflete as limitações de capacidade, determinando assim o início e término para cada tarefa em cada centro de trabalho. Nesse cenário, se utiliza uma abordagem chamada sequenciamento de sistemas de capacidade finita (FCS).

Segundo Fernandes (2006) existem algumas abordagens utilizadas em FCS, por exemplo, a de sequenciamento baseado em trabalho (job-based), a qual consiste em carregar a tarefa de prioridade mais alta nos centros de trabalho e posteriormente agendar o trabalho com a próxima prioridade e assim por diante.

\subsection{Modelos de sequenciamento da produção}

Existem diversos problemas de scheduling, os quais são caracterizados pela relação entre o número de máquinas (recursos), ordens de produção (jobs) e operações (ROSS, HART e CORNE, 2005). O Job Shop Scheduling (JSS) é um desses problemas. Fattahi, Mehrabad, e Jolai (2007) descrevem que nesse problema o objetivo é alocar $n$ ordens de produção em $m$ máquinas, no qual cada ordem possui um conjunto de operações com roteiro pré-determinado, além disso é preciso satisfazer dois tipos de restrições: a sequência de máquinas que um job tem que visitar é fixa; a outra é que a máquina processa um job por vez. A Figura 1 ilustra esse tipo de sequenciamento.

Figura 1 - Exemplo de sequenciamento em um sistema job shop. 


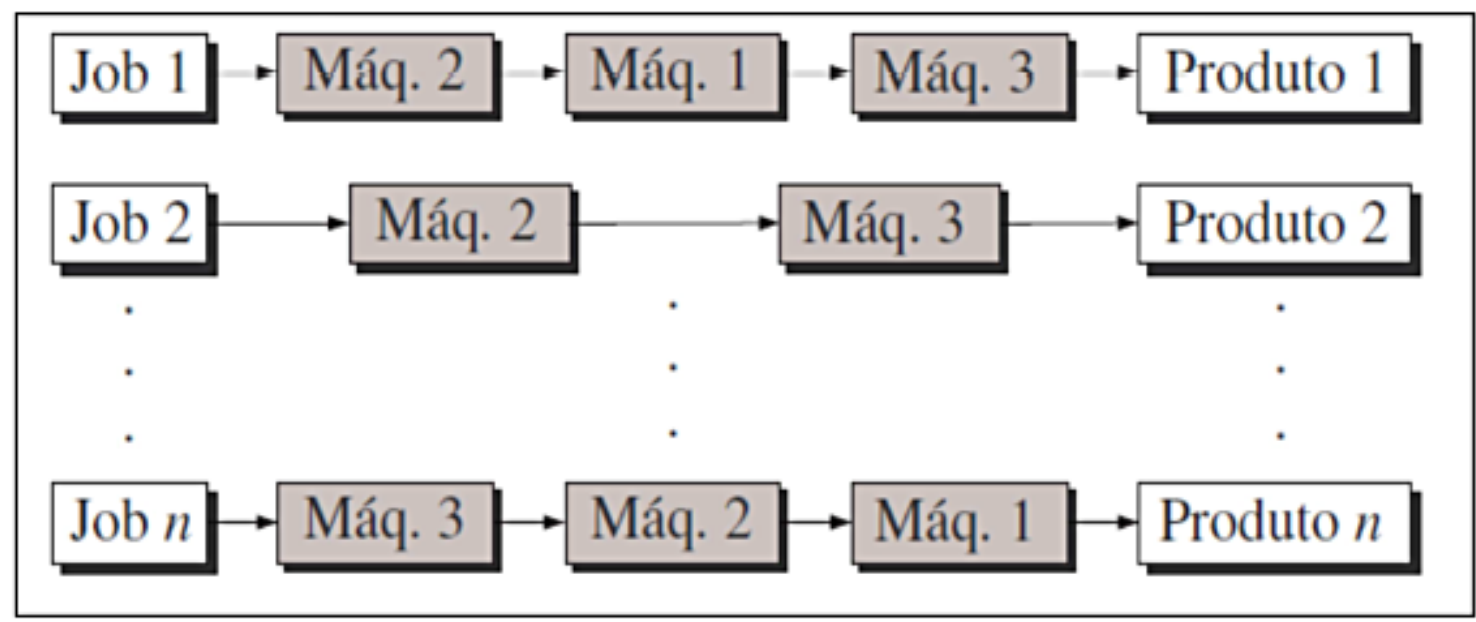

Fonte - Fattahi, Mehrabad e Jolai (2007).

Conforme Lustosa et al. (2008) o sequenciamento para JSS apresenta elevada complexidade. Ao pensar na solução desse tipo de problema deve-se levarse-á em conta alguns aspectos, como:

- Número de máquinas envolvidas;

- Roteiros das ordens de produção;

- Regime de chegada das ordens; e

- A variabilidade dos tempos de processamento.

\section{METODOLOGIA}

Lakatos e Marconi (1992, p. 105) afirmam que a especificação da metodologia da pesquisa é a que abrange maior número de itens, pois responde em um só tempo, as seguintes questões: como?, com quê?, onde?, e quanto?. Conforme Gil (2002) a pesquisa científica é um procedimento racional e sistêmico que objetiva promover respostas a essas questões. Segundo esse mesmo autor, esclarecer as etapas da pesquisa e o sequenciamento delas é importante para a avaliação do processo de pesquisa. Nesse sentido, a Figura 2 apresenta o detalhamento das etapas e atividades que foram desenvolvidas durante a presente pesquisa.

Figura 2 - Detalhamento das etapas e atividades da presente pesquisa. 


\section{1 - Definição do contexto e propósito}

\section{2 - Definição da estrutura conceitual-teórica}

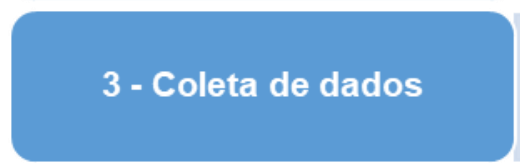

4 - Análise dos dadose desenvolvimento do propósito

5 - Avaliação e discussão dos resultados
- Diagnosticar a situação atual da organização;

- Definir tema e interessados;

- Delimitar o problema.

- Mapear literatura;

- Delinear ideias e proposições;

- Classificar a pesquisa.

- Definir técnicas de coleta de dados;

- Registrar dados.

- Tabular dados;

- Analisar dados;

- Desenvolver propósito

- Avaliar resultados;

- Prover estrutura para reaplicação;

- Desenhar implicações teóricas e práticas;

- Redigir relatório

Fonte: Adaptado de Miguel et al. (2018).

Conforme a Figura 2 a estrutura da pesquisa dividiu-se em 5 etapas, na primeira, definição do contexto e propósito, realizou-se o diagnóstico da empresa, onde foram apresentadas as problemáticas identificadas, posteriormente houve a delimitação do tema. Já na segunda etapa, definição da estrutura conceitual-teórica, o objetivo foi realizar uma revisão bibliográfica sobre o tema visando a compreensão efetiva sobre o assunto. Em contrapartida na terceira etapa ocorreu a coleta de dados, o foco foi a elaboração da cronoanálise dos subprodutos. Na quarta etapa, análise dos dados e desenvolvimento do propósito, aconteceu o desenvolvimento do sequenciamento da produção. Finalmente na quinta etapa, avaliação e discussão dos resultados, realizou-se uma análise dos resultados obtidos, as limitações da pesquisa e recomendações para estudos futuros também foram relatadas.

\subsection{Definição da Estrutura Conceitual-Teórica}

Segundo Zanella (2013) após a definição do tema é preciso conhecer o que os autores dizem sobre ele, para isso é necessário fazer um levantamento de dados, esse levantamento inclui a pesquisa bibliográfica. Para a realização desta pesquisa utilizou-se o método científico revisão bibliográfica sistemática (RBS). 
Conforto, Amaral e Silva (2011) descrevem a RBS como um método que auxilia no mapeamento e seleção de estudos publicados mais relevantes sobre um tema específico, possibilitando assim a elaboração de um resumo sobre o conhecimento já existente. O modelo utilizado para a condução da RBS possui três fases, são elas: entrada, processamento e saídas.

$\mathrm{Na}$ entrada, definiu-se os elementos das 8 etapas, o Quadro 3 apresenta um resumo dessa fase.

Já na fase do processamento, os artigos pré-selecionados foram analisados e processados nos três filtros descritos no quadro acima. Por fim, tem-se a terceira fase, onde ocorre a categorização dos artigos selecionados. Os resultados da RBS em relação as bases de dados acessadas, quantidade de artigos encontrados, selecionados e excluídos podem ser observados no Quadro 4.

Após a finalização da atividade de mapeamento da literatura é realizada a classificação do conhecimento e da pesquisa. Nesse estudo o conhecimento apresentado é de caráter científico, pois conforme Zanella (2013, p. 15) esse conhecimento procura conhecer além do fenômeno e resulta de uma investigação metódica e sistemática da realidade, buscando as causas dos fatos e as leis que os regem. Em consonância com os procedimentos intelectuais e técnicos adotados nesta pesquisa, pode-se classificar o método usado como indutivo, visto que segundo Lakatos e Marconi (1992), nesse método o conhecimento é embasado na experiência, e a generalização deriva de observações da realidade.

Quadro 3 - Resumo da fase entrada

\begin{tabular}{|l|l|}
\hline PROBLEMA & $\begin{array}{l}\text { Como propor um sequenciamento dos subprodutos de uma } \\
\text { empresa com estratégia make to order? }\end{array}$ \\
\hline OBJETIVOS & $\begin{array}{l}\text { Analisar estudos, publicações e relatos sobre sequenciamento da } \\
\text { produção por meio de uma revisão bibliográfica sistemática (RBS). } \\
\text { Com o propósito de identificar elementos que compõem as } \\
\text { abordagens sobre esse tema }\end{array}$ \\
\hline FONTES PRIMÁRIAS & $\begin{array}{l}\text { Slack, Chambers e Johnston (2009), Russomano (2000) e Tubino } \\
\text { (2009) }\end{array}$ \\
\hline PALAVRAS-CHAVE & $\begin{array}{l}\text { Scheduling. Job shop. Make to order. Finite capacity systems. Gantt } \\
\text { chart }\end{array}$ \\
\hline STRINGS DE BUSCA & "Scheduling" AND “job shop" AND "make to order" \\
\hline CRITÉRIOS INCLUSÃO & $\begin{array}{l}\text { Os artigos deverão conter assuntos relacionados as palavras- } \\
\text { chaves; a publicação não poder ser apenas um resumo; e é crucial } \\
\text { ter acesso completo ao artigo }\end{array}$ \\
\hline $\begin{array}{l}\text { CRITÉRIOS } \\
\text { QUALIFICAÇÃO }\end{array} \quad$ DE & $\begin{array}{l}\text { Ter caráter descritivo; a pesquisa deverá ser classificada como } \\
\text { bibliográfica e/ou pesquisa-ação; e possuir no mínimo 10 citações }\end{array}$ \\
\hline
\end{tabular}




\begin{tabular}{|l|l|}
\hline MÉTODO E FERRAMENTAS & $\begin{array}{l}\text { Processar os artigos nos três filtros e armazenar os resultados em } \\
\text { uma planilha no programa Microsoft Excel. } 1^{\circ} \text { filtro }-0 \text { artigo deve } \\
\text { abordar sobre sequenciamento da produção; } 2^{\circ} \text { filtro }-0 \text { artigo } \\
\text { precisa apresentar assuntos relacionados a job shop e make to } \\
\text { order. } 3^{\circ} \text { filtro }-0 \text { artigo deverá atender aos critérios de inclusão e } \\
\text { qualificação }\end{array}$ \\
\hline
\end{tabular}

Quadro 4 - Resultado da revisão bibliográfica sistemática

\begin{tabular}{|l|l|}
\hline Base de dados & Emerald Insight e Web of science (thomson reuters) \\
\hline Palavras-chave & $\begin{array}{l}\text { Scheduling. Job shop. Make to order. Finite capacity systems. } \\
\text { Gantt chart }\end{array}$ \\
\hline $\begin{array}{l}\text { Total bruto de artigos } \\
\text { encontrados }\end{array}$ & 152 \\
\hline Total de artigos selecionados & $\begin{array}{l}\text { 5, são eles: Novas, 2019; Chung-Hsing, 2000; Pinedo, 2008; } \\
\text { Phanden, Jain e Verma, 2012; e Ross, Hart e Corne, 2005. }\end{array}$ \\
\hline
\end{tabular}

Em relação a classificação da presente pesquisa e no que concerne à sua natureza, pode-se denominá-la como aplicada. Silva e Menezes (2015, p.20) afirmam que esse tipo de pesquisa objetiva gerar conhecimentos para aplicação prática e dirigidos à solução de problemas específicos, envolvendo verdades e interesses locais.

Do ponto de vista da forma de abordagem do problema, a pesquisa é caracterizada como combinada de triangulação. Segundo Paranhos (et al., 2016) as técnicas quantitativas e qualitativas têm potencialidades e limitações, elas são utilizadas com propósitos diferentes. A vantagem da integração consiste em retirar o melhor de cada uma para responder uma questão específica. A qualitativa foi utilizada para interpretar o ambiente em que a problemática está inserida por meio de uma observação individual de campo participante, e a quantitativa na mensuração dos tempos de processamento dos subprodutos. Segundo seus objetivos considera-se como pesquisa descritiva, ao passo que o estudo visa descrever algumas problemáticas geradas na ausência de um sequenciamento. De acordo com os procedimentos técnicos utilizados, o estudo classifica-se como pesquisa bibliográfica, visto que é elaborada a partir de estudos já publicados; e também como pesquisa-ação, onde o pesquisador faz parte da equipe e está envolvido no processo.

\subsection{Coleta de Dados}


A coleta de dados ocorreu no período entre julho e setembro de 2019. Sendo que a cronoanálise dos subprodutos foi realizada no mês de agosto; já os tempos de conferência das ordens foram coletados no mês de setembro. O tempo total de processamento dos subprodutos foi o resultado da soma entre o tempo gasto com setup para as montagens dos subprodutos e o tempo total de fabricação dos mesmos. Para a coleta utilizou-se a técnica de observação participante, de acordo com Lakatos e Marconi (1992) essa técnica consiste em analisar fenômenos e fatos que se deseja estudar. Diante disso, diversos aspectos foram observados, como: processos produtivos; critérios e prioridades de produção e tempo de processamento das ordens. Os registros dos dados ocorreram por meio de fotografias, filmagens e diário de campo. Os instrumentos utilizados foram: cronômetro para realizar a cronoanálise e planilhas do programa Microsoft Excel para registrar os dados e gerar gráficos. A duração do tempo em que os dados são coletados é do tipo corte-transversal, uma vez que a coleta ocorre em um só momento, pretendendo descrever e analisar os estados das variáveis em um momento específico.

\section{RESULTADOS}

A Cristófoli e Camacho Equipamento Hospitalar Ltda é especializada na fabricação de autoclaves hospitalares e está localizada no município de Campo Mourão/PR. É considerada de pequeno porte, atua no setor metal-mecânico, e atualmente encontra-se incubada a outra organização, a Cristófoli Equipamentos de Biossegurança.

A autoclave produzida pela Cristófoli e Camacho realiza a esterilização de objetos por meio do alto-vácuo pulsante e vapor saturado sob pressão e secagem, esse equipamento é composto por várias matérias-primas e subprodutos. Ela possui capacidade de 100L e uma porta, mas podem ocorrer modificações no projeto visando atender as especificações dos clientes. As alterações realizadas estão relacionadas a quantidade de litros, $100 \mathrm{~L}$ a $300 \mathrm{~L}$; e ao número de portas, uma ou duas.

A empresa já fabricou autoclaves de 100L, 200L e 300L, todas com duas portas, porém as estruturas desses produtos, bem como os processos de montagem 
e funcionamento não estão padronizados, pois encontram-se na fase de projeto, na qual são analisados e testados.

Diante do exposto, a pesquisa limitou-se em abordar apenas o modelo $100 \mathrm{~L}$ com uma porta (CH100 1P).

\subsection{Características do Processo Produtivo}

O início da produção acontece mediante pedido de venda, o que caracteriza a estratégia de negócio como make to order, ou seja, produz sob encomenda.

Após a abertura do pedido de venda o departamento PCP da Biossegurança emite uma ordem para cada subproduto que compõe a autoclave, são 22 subprodutos. O processo de emissão das ordens não é orientado por uma sequência preestabelecida, tampouco consideram restrições de precedências. Além do mais, as emissões ocorrem em períodos e dias diferentes. Os subprodutos podem ser classificados em dois níveis, no nível 2 estão os subprodutos antecessores aos subprodutos do nível 1, isto é, para produzir os subprodutos do nível 1 é imprescindível que os subprodutos do nível 2 estejam finalizados.

Posteriormente o setor de almoxarifado da matriz separa as matériasprimas

para cada ordem e envia as mesmas para a Camacho, onde são processadas. Finalizando a montagem dos subprodutos, esses são encaminhados ao almoxarifado da Biossegurança.

Seguidamente o PCP emite uma ordem geral para a montagem da autoclave, a qual contém os 22 subprodutos produzidos anteriormente e outros componentes. Essa ordem é enviada para a Camacho, na qual é realizada a fabricação, embalagem e expedição do produto final. A Figura 3 apresenta um fluxograma dos processos descritos acima.

Figura 3 - Fluxograma dos processos produtivos 


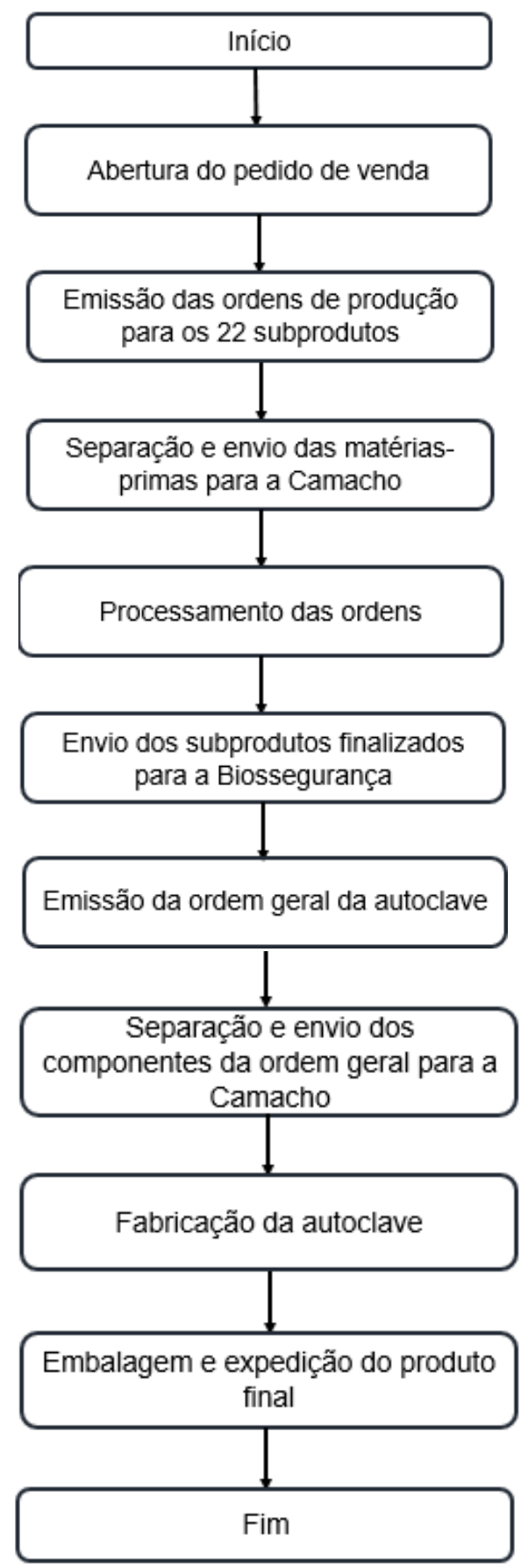

\subsection{Problemática}

Fonte: Dados da pesquisa (2019).

A principal problemática identificada no ambiente produtivo da Cristófoli e Camacho foi o elevado tempo de processamento das ordens dos subprodutos. Ao analisar a causa raiz do problema, contatou-se que alguns fatores influenciavam diretamente, são eles: 
- Inexistência de um processo de sequenciamento das ordens dos subprodutos que considerasse as restrições de precedências.

- Ausência de um setor responsável pelo recebimento, conferência e liberação das ordens.

- Ausência de um setor responsável pelo recebimento.

Em relação ao sequenciamento das ordens, durante 0 estudo sobre 0 equipamento foi possível identificar a existência de restrições de precedências entre os subprodutos, sendo que durante a emissão, liberação e processamento das ordens essas restrições não eram consideradas, o que acarretava em diversas disfunções, como:

- Paradas não planejadas durante a montagem em consequência da falta de subprodutos antecessores.

- Falta de matérias-primas para os subprodutos do nível 2, devido a abertura e separação das ordens do nível 1 acontecerem antes das ordens do nível 2, visto que algumas matérias-primas são comuns entre alguns subprodutos.

- Além de elevar o tempo de produção, e inatividade dos colaboradores, já que os mesmos interrompiam o processo para solucionar as disfunções identificadas.

Quanto ao recebimento, liberação e conferência das ordens, notou-se que os responsáveis por essas atividades eram os colaboradores da produção. Logo, a identificação de problemas relacionados a falta de componentes, envio incorreto de peças e não conformidades com materiais ocorria durante a montagem. Diante disso os colaboradores paravam a produção e solucionavam essas disfunções, o tempo de resolução era relativamente alto, podendo demorar horas ou até mesmo dias.

Sobre o fator organização do ambiente produtivo, foi verificado que os espaços destinados a produção, armazenamento de materiais e realização de testes não estavam sendo aproveitados de maneira efetiva, e nem havia organização nos mesmos. Perante esse cenário o tempo para encontrar uma ferramenta, localizar uma ordem de produção e identificar um equipamento para teste era elevado. 
Todas essas variáveis interferiam nos resultados da produção. À vista disso, foi realizado um levantamento das possíveis soluções que amenizassem essas problemáticas e reduzisse o tempo total de processamento dos subprodutos.

Diante desse cenário, constatou-se que a alternativa mais viável e que colabora em diversos aspectos para empresa é o desenvolvimento de um sequenciamento da produção voltado para os 22 subprodutos que compõe a autoclave $\mathrm{CH} 100$ 1P. A partir de um sequenciamento da produção que considere as restrições de precedências o departamento PCP poderá realizar um planejamento assertivo das ordens e o almoxarifado conduzirá a separação dos materiais de maneira mais efetiva, respeitando as prioridades; consequentemente reduzindo a falta de materiais. Também servirá para a organização das atividades produtivas em paralelo com a implantação de um setor de recebimento, conferência e liberação das ordens, visando a obtenção de um fluxo eficiente, isto é, sem paradas não planejadas.

Após apresentar o diagnóstico da situação atual da empresa ao diretor e evidenciar que diversos problemas estão relacionados a falta de um sequenciamento da produção e como sua aplicabilidade otimizaria os resultados gerenciais, decidiu-se que o desenvolvimento de um estudo na área de Planejamento e Controle da Produção voltado à atividade de sequenciamento da produção é viável e relevante.

\subsection{Descrição dos parâmetros do problema}

A seguir serão descritos os parâmetros que envolvem o problema de sequenciamento, ou seja, a descrição dos recursos, dos subprodutos, apresentação do tempo total de processamento dos mesmos, das restrições de precedências e rota de cada subproduto.

Os subprodutos circulam entre os postos de trabalho caracterizando o layout da fábrica como do tipo funcional (por processos), no qual há um agrupamento no mesmo local de operações semelhantes.

As montagens dos 22 subprodutos são realizadas manualmente por 3 colaboradores, 2 voltados para os processos de montagem pneumática e outro para 
os processos de solda. Cada colaborador processa um subproduto por vez, tal como cada subproduto possui uma rota preestabelecida. A cerca disso, o sistema de manufatura é classificado como job shop. O tempo total de processamento dos subprodutos são apresentados na Tabela 1 , enquanto que as restrições de precedências podem ser observadas na Figura 4.

Tabela 1 - Tempo total de processamento dos subprodutos.

\begin{tabular}{clc}
\hline ATIVIDADE & \multicolumn{1}{c}{ DESCRIÇÃO DO SUBPRODUTO } & $\begin{array}{c}\text { TEMPO DE } \\
\text { PROCESSAMENTO }\end{array}$ \\
\hline A & CONJUNTO VÁLVULA DE SEGURANÇA DO GERADOR M1 & $00: 18: 43$ \\
B & CONJUNTO TRANSMISSOR DE PRESSÃO CÂMARA INTERNA P1 & $00: 15: 38$ \\
C & CONJUNTO PURGADOR CÂMARA EXTERNA CH100 M1 & $00: 32: 27$ \\
D & CONJUNTO BLOCO MANIFOLD M1 1P D32 & $01: 10: 22$ \\
E & CONJUNTO PLACA PAINEL ELÉTRICO & $01: 16: 00$ \\
F & CONJUNTO ENTRADA VAPOR CÂMARA INTERNA CH100 M1 & $01: 17: 07$ \\
G & CONJUNTO DESCARGA CÂMARA INTERNA CH100 M1 & $01: 18: 54$ \\
H & CONJUNTO DESCARGA GERADOR CH100 M1 & $00: 44: 11$ \\
I & CONJUNTO ENTRADA DE ÁGUA BOMBA DE VÁCUO M1 & $00: 12: 15$ \\
J & CONJUNTO ENTRADA DE ÁGUA BOMBA DE ÁGUA M1 & $00: 28: 56$ \\
K & CONJUNTO PORTA LADO A (SUJO) IMH 3,5" CH100 M1 & $57: 00: 00$ \\
L & CONJUNTO EVAPORADOR M1 & $00: 50: 00$ \\
M & MONTAGEM ATUADOR TAMPA D32 & $00: 26: 12$ \\
N & TAMPA LADO (A) SUJO M1 1P & $04: 46: 00$ \\
O & CUBA CH100 1P M1 & $00: 50: 00$ \\
P & REGIME CH100 M1 & $00: 14: 32$ \\
Q & CONJUNTO DA BOMBA DE VÁCUO CH100 M1 & $00: 51: 47$ \\
R & CONJUNTO TRANSMISSOR DE PRESSÃO CÂMARA EXTERNA & $00: 10: 56$ \\
S & ISOLAMENTO TÉRMICO CH100 1P & $02: 30: 00$ \\
T & PAINEL ELÉTRICO CH100 M1 PORTA DELTA & $06: 42: 00$ \\
U & VÁLVULA RETENÇÃO LATÃO COM MOLA & $00: 06: 00$ \\
V & CONJUNTO REGIME CH100 INOX 304 & $00: 07: 32$ \\
\hline
\end{tabular}

Fonte: Dados da pesquisa (2019).

Figura 4 - Relação de precedência entre os subprodutos apresentados na Tabela 1 


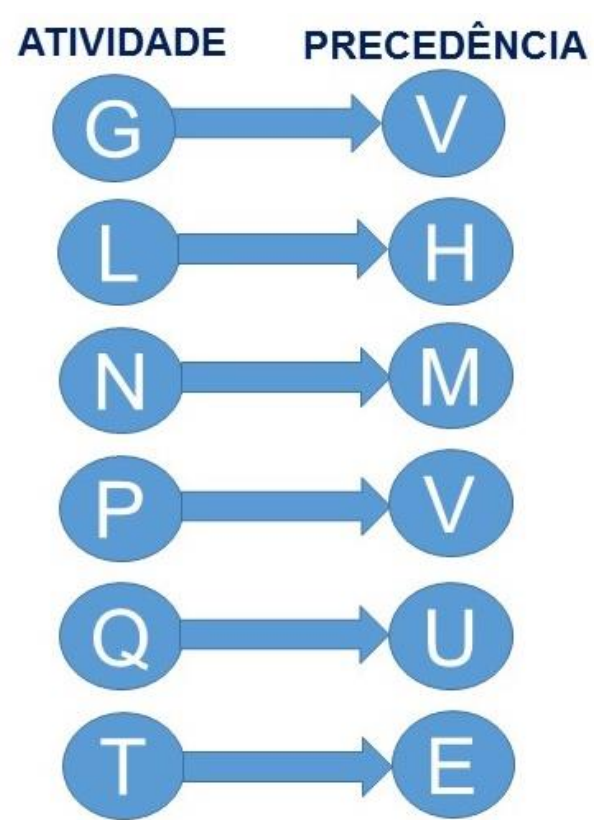

Fonte: Dados da pesquisa (2019).

A Figura 5 apresenta a rota de cada subproduto nos centros de trabalho, em que C1P é o centro onde estão localizados os colaboradores que realizam as montagens pneumáticas e o C2S é o centro de soldagem, tendo apenas um colaborador.

Figura 5 - Rota de cada subproduto nos centros de trabalho.

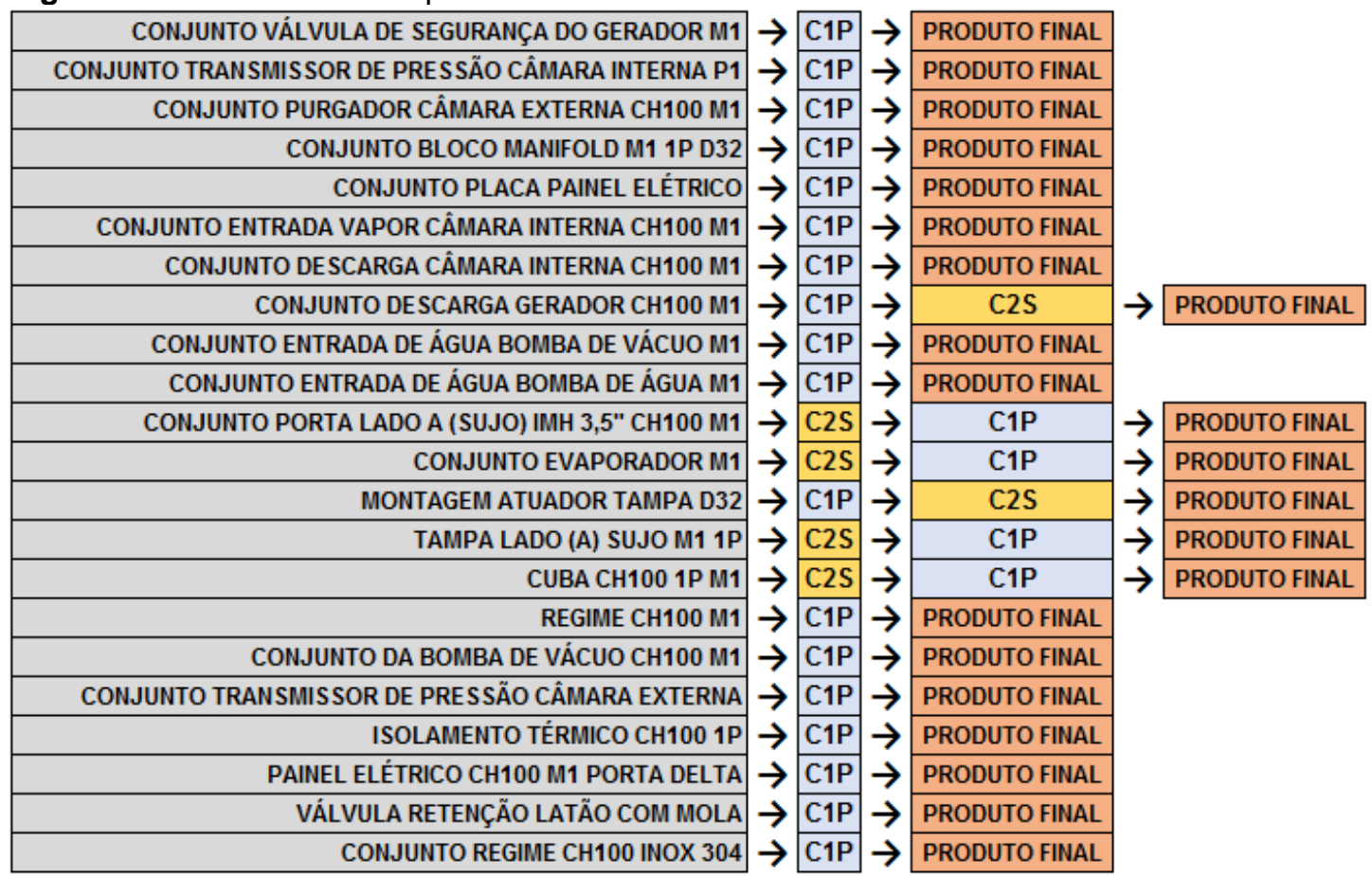

Fonte: Dados da pesquisa (2019).

\subsection{Proposta de Sequenciamento}


Uma análise sobre as regras de sequenciamento e dos objetivos da organização foi realizada visando a elaboração da proposta de sequenciamento dos subprodutos. O principal objetivo da empresa é reduzir o tempo de processamento dos subprodutos, logo o sequenciamento deve ser realizado visando atender tal objetivo. Nesse sentido, após um estudo sobre as regras de sequenciamento, constatou-se que a regra mais adequada para realização do sequenciamento é a MTP em consonância com as restrições de precedências. Como abordado anteriormente, essa regra objetiva sequenciar os lotes de produção segundo os menores tempos de processamento. À vista disso, ao sequenciar as atividades respeitou-se a relação de precedência, tendo como exemplo, a atividade $\mathrm{G}$ somente será realizada se a atividade $\mathrm{V}$ estiver concluída.

Em relação as outras regras de sequenciamento, a regra PEPS não pode ser aplicada porque durante o período que se realizou a cronoanálise a empresa ainda não possuía um setor de recebimento e conferência das ordens. Logo, não existia nenhum controle sobre a chegada das ordens de produção. Já a regra MDE não foi utilizada, pois também nesse período de pesquisa não existiam datas definidas para as entregas das ordens. Uma vez que, para iniciar a montagem da autoclave, todos os subprodutos precisam estar finalizados, ou seja, todos têm a mesma data de entrega. No que diz respeito a prioridade dos pedidos, é a mesma para todos. Diante disso a regra IPI não pode ser aplicada. Também optou-se por não utilizar as regras ICR, IFO e IFA, posto que essas são voltadas para sistemas informatizados, o que não é a realidade atual da empresa. Sendo assim, a Figura 6 ilustra a proposta de sequenciamento para os subprodutos que compõem a autoclave $\mathrm{CH} 1001 \mathrm{P}$.

Após a elaboração do sequenciamento, o mesmo foi apresentado a direção e ao departamento PCP, ambos confirmaram que o estudo contribui para o planejamento das ordens e também é útil para o departamento almoxarifado, visto que os colaboradores serão orientados a enviar as ordens de acordo com 0 sequenciamento desenvolvido, visando reduzir problemas com falta de materiais e paradas não planejadas na produção.

Figura 6 - Proposta de sequenciamento dos subprodutos segundo a regra MTP 


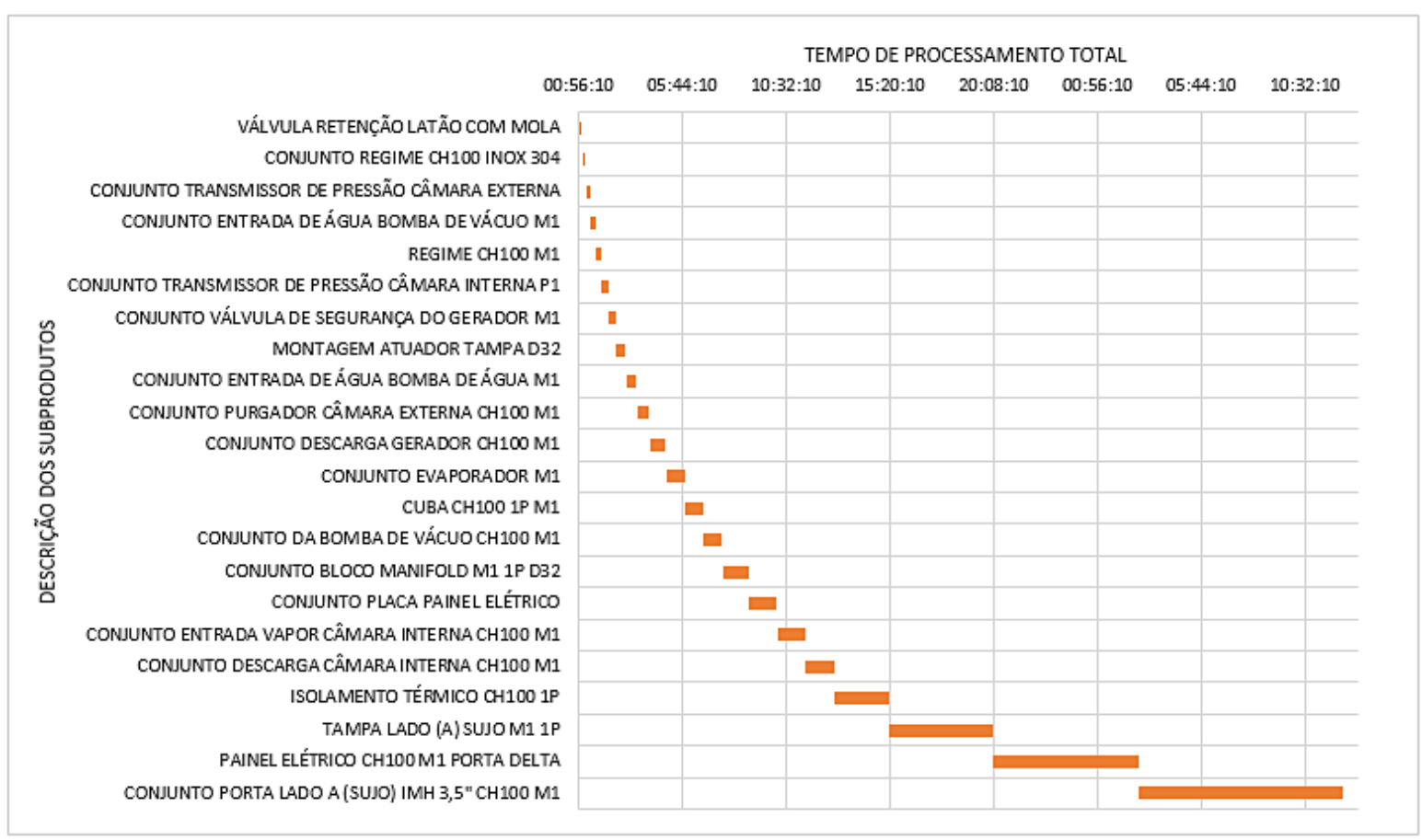

Fonte: Elaborado pela autora (2019).

Para a realização da cronoanálise foram necessários vários dias dedicados apenas a essa atividade, já que durante as montagens houve diversas paradas devido à falta de matérias-primas e de subprodutos antecessores, em alguns casos a fabricação de alguns itens ficou parada mais de uma semana, pois foi necessário o PCP emitir ordens de subprodutos antecessores, os colaboradores realizarem as montagens para então dar continuidade a produção dos subprodutos que ficaram parados. Analisando o tempo total de processamento proposto pelo sequenciamento, 34:35:32, é possível observar que o tempo de produção é reduzido, visto que para a produção de 22 subprodutos foram necessários 13 dias, cerca de 312 horas, 104 horas por colaborador. Solucionando problemas com falta de matérias-primas, temos uma redução de $89 \%$ do tempo total de processamentos dos subprodutos.

A partir do resultado do sequenciamento notou-se que a produção de tampas, painéis e portas demandam muito tempo da produção, diante disso o engenheiro mecânico realizou uma análise do processo de montagem desses itens e está alterando os projetos com o objetivo de reduzir os tempos de processamento e tornar a produção mais prática. Posteriormente também será feita uma análise dos outros subprodutos visando melhorias. 
Portanto, conclui-se que a elaboração do sequenciamento dos subprodutos foi de grande relevância para todos os envolvidos, alguns resultados positivos relacionados ao planejamento de ordens futuras já foram notados, bem como a conscientização dos colaboradores sobre a importância de seguir a proposta apresentada.

\subsection{Organização do processo e ambiente}

Em relação a organização, realizou-se uma reunião com os colaboradores, na qual discutiu-se a importância de manter os ambientes limpos e organizados. Nessa mesma reunião eles puderam expressar opiniões e sugestões de melhorias que foram anotadas e utilizadas no desenvolvimento de um plano de ação, que abordou as atividades e serem realizadas visando a organização e limpeza da empresa. Esse plano foi apresentado e então deu-se início a execução de suas etapas.

Na primeira etapa houve a separação e descarte de materiais que não tinham mais utilidade para os processos. Posteriormente ocorreu a limpeza dos ambientes, e em seguida os colaboradores organizaram todos os objetos de maneira que facilitasse a realização dos trabalhos.

Ao longo da realização das atividades notou-se resistência por alguns colaboradores quanto a aceitação das mudanças realizadas, principalmente em relação aos descartes de materiais. Entretanto, no decorrer dos dias eles notaram resultados positivos e então se engajaram.

Durante alguns feedbacks dos colaboradores já pode-se verificar algumas melhorias, por exemplo, a redução do tempo para procurar ferramentas, uma vez que atualmente cada setor tem à disposição um painel com todas as ferramentas necessárias para realizar as atividades, facilitando assim a identificação e reduzindo a movimentação durante o trabalho.

No ambiente produtivo as ferramentas e equipamentos utilizados nos processos ficavam espalhados pela produção, essa situação gerava um aumento no tempo de processamento, visto que os colaboradores demoravam para encontrá-los. Outra questão era a dificuldade em localizar os materiais utilizados nas montagens, pois não existia um local em que estes fossem recebidos e armazenados até a 
liberação para a produção, logo os materiais se encontravam no chão da produção sem nenhuma identificação ou precaução para que não fossem utilizados para outros fins ao qual não estivessem destinados. À vista disso algumas melhorias foram desenvolvidas, como os painéis de ferramentas, também houve uma mudança no layout, onde o departamento administrativo foi transferido para outra sala distante da produção, e o setor de solda foi realocado para esse espaço disponível. Essa mudança surtiu ótimos efeitos, quanto a redução de ruídos e a exposição dos colaboradores aos raios emitidos durante a soldagem. Outra mudança foi a disponibilização de carrinhos identificados e com todos os subprodutos e materiais necessários para a montagem do equipamento, diminuindo assim desperdícios relacionados a transportes, movimentação dos colaboradores, além de facilitar o acesso aos recursos e impossibilitar o uso indevido de materiais.

Com relação ao local de armazenamento de materiais, o mesmo se encontrava com um excesso de prateleiras e materiais que já não eram mais utilizados pela produção e outros departamentos, além disso a movimentação dentro desse ambiente era muito limitada. Sendo assim, algumas prateleiras foram retiradas com o objetivo de viabilizar a movimentação eficiente das pessoas; as prateleiras que permaneceram no local foram identificadas com etiquetas, separando ordens a serem montadas de produtos acabados e materiais que são utilizados em protótipos. Parte desse espaço também foi destinado ao setor de recebimento e conferência das ordens de produção. Ao final desse remanejamento pode-se observar uma melhora significativa quanto à localização e identificação dos objetos e movimentação de pessoas.

Por fim, no que se refere ao ambiente de testes, antes os equipamentos e materiais utilizados se encontravam espalhados pelo ambiente produtivo, administrativo e de armazenamento, dificultando dessa forma o acesso a eles. Logo, foi notória a necessidade de organização, diante disso houve uma seleção de todos os equipamentos e materiais utilizados nos testes, os mesmos foram organizados e armazenados em uma prateleira na área onde os testes são realizados, proporcionando fácil identificação e acesso. 


\subsection{Proposta de melhoria no recebimento, conferência e liberação das ordens de produção}

Quanto ao recebimento e conferência das ordens a responsabilidade era dos colaboradores da produção. Esse cenário gerava desperdícios, como: espera, movimentação e transporte. A Figura 7 ilustra um fluxograma de como o processo ocorria.

Figura 7 - Fluxograma do processo de recebimento e conferência das ordens antes de implantar o setor de recebimento

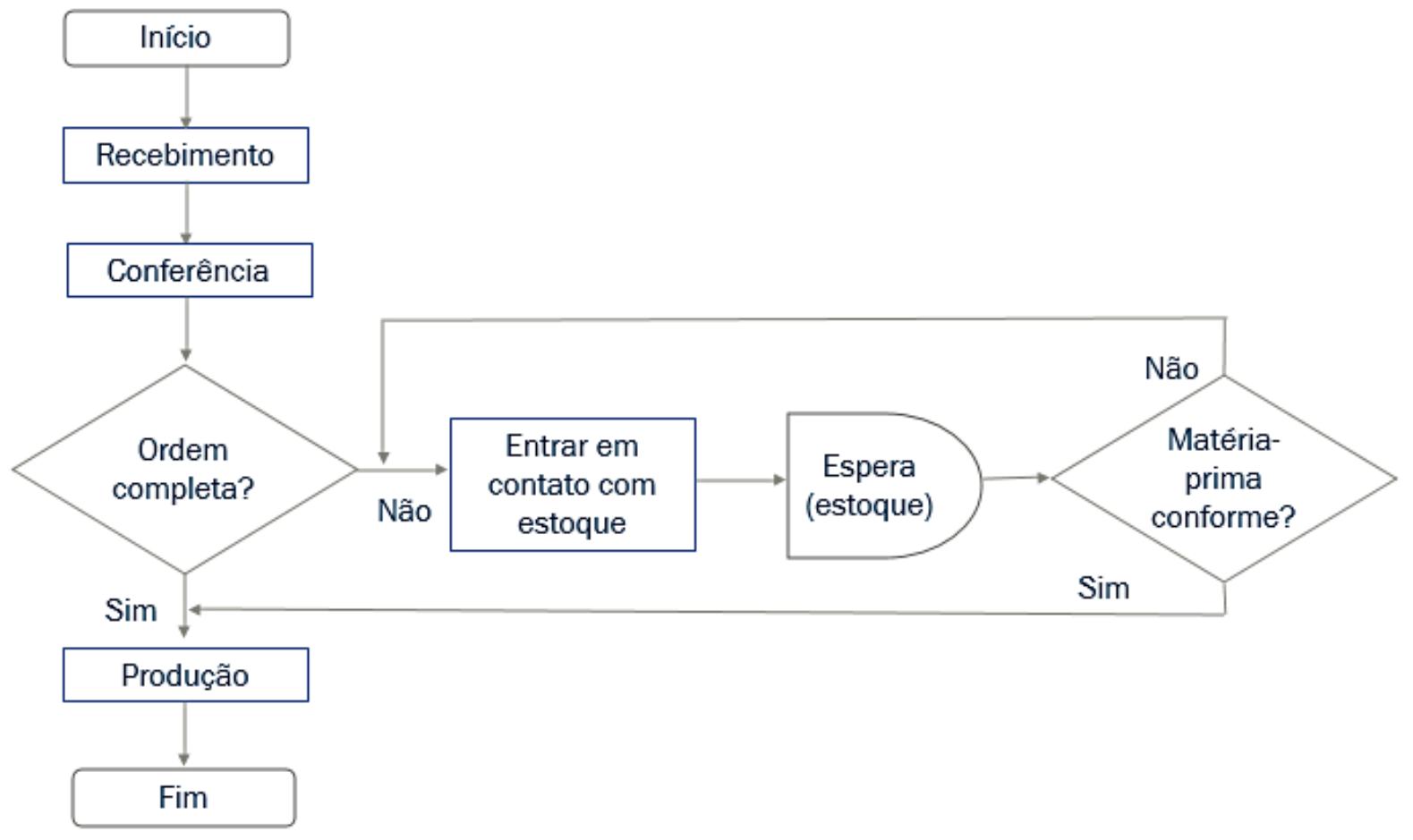

Fonte: Elaborado pela autora (2019).

Após o consentimento da direção, a pesquisadora deu início a implantação do processo de recebimento e conferência das ordens. Atualmente as ordens são recebidas e conferidas antes de serem direcionadas à produção, quando se identifica falta de matéria-prima o responsável pelo setor de suprimentos recebe uma planilha via e-mail com uma lista dos itens que vieram faltando, posteriormente os mesmos são separados e encaminhados à produção. Dessa maneira a ordem de produção só é liberada quando se encontra completa. Durante esse período de recebimento e conferência os colaboradores estão exclusivamente realizando as 
montagens. Após a implantação desse processo constatou-se uma redução da ociosidade, movimentação, transporte e tempo de processamento. Além de contribuir para a revisão dos componentes das ordens, já que foram identificadas várias disfunções relacionadas as composições das ordens. A Tabela 2 apresenta os tempos que o atual setor de recebimento leva para concluir a conferência de algumas ordens.

Tabela 2 - Tempo atual do setor para conferência de ordens

\begin{tabular}{lc}
\multicolumn{1}{c}{ NOME DO CONJUNTO } & $\begin{array}{c}\text { TEMPO DE } \\
\text { CONFERÊNCIA }\end{array}$ \\
\hline CONJUNTO TRANSMISSOR DE PRESSÃO CÂMARA INTERNA P1 & $00: 10: 31$ \\
CONJUNTO PLACA PAINEL ELÉTRICO & $00: 15: 00$ \\
CONJUNTO DESCARGA CÂMARA INTERNA CH100 M1 & $00: 40: 00$ \\
CONJUNTO PORTA LADO A (SUJO) IMH 3,5" CH100 M1 & $00: 24: 00$ \\
CONJUNTO EVAPORADOR M1 & $00: 20: 00$ \\
TAMPA LADO (A) SUJO M1 2P & $00: 26: 00$ \\
CUBA CH100 1P M1 & $00: 30: 00$ \\
PAINEL ELÉTRICO CH100 M1 PORTA DELTA & $02: 26: 00$ \\
CONJUNTO REGIME CH100 INOX 304 & $00: 15: 32$ \\
\hline
\end{tabular}

Fonte: Dados da pesquisa (2019).

Diante disso, fica evidente que futuramente o tempo de processamento dos subprodutos irá reduzir, uma vez que os colaboradores não irão mais realizar conferências ou precisarão resolver problemas sobre falta de componentes, essas responsabilidades serão exclusivamente do setor implantado.

As ações desenvolvidas objetivando melhorias relacionadas à organização dos ambientes e a implantação de um setor que realizasse o recebimento e conferência das ordens foram fundamentais para o desenvolvimento do sequenciamento e servirão como apoio na aplicação do sequenciamento proposto.

\section{CONSIDERAÇÕES FINAIS}

Ao iniciar a pesquisa constatou-se a seguinte problemática: a inexistência de um sistema que realizasse a ordenação das ordens de produção dos subprodutos que compõe o principal produto comercializado pela empresa. À vista disso 
identificou-se a relevância no desenvolvimento de uma pesquisa sobre sequenciamento da produção.

Sendo assim, a pesquisa teve como objetivo geral apresentar uma proposta de sequenciamento dos subprodutos que compõe o principal produto da empresa. Constatou-se que o objetivo foi atendido, dado que ao final do estudo conseguiu-se apresentar uma proposta de sequenciamento.

Em relação às limitações da pesquisa, houve dificuldade em obter um histórico sobre os resultados da empresa, como a capacidade produtiva e tempo total de processamento dos subprodutos. Quanto ao desenvolvimento dos resultados, no início o objetivo era realizar uma comparação entre os resultados obtidos por cada regra de sequenciamento apresentadas no referencial teórico, porém isso não foi possível, porque a empresa ainda não definiu datas de entregas para as ordens, já que atualmente todos os subprodutos necessariamente precisam estar prontos no mesmo dia. Outro obstáculo sobre a aplicação dessas regras está relacionado a falta de controle de chegadas das ordens, o que também afetou os resultados planejados anteriormente. A limitação de tempo também influenciou, uma vez que a cronoanálise foi realizada apenas uma vez para cada conjunto, é fato que após a implantação de algumas melhorias os tempos de processamento seriam modificados.

Quanto às recomendações para futuras pesquisas, aconselha-se realizar um estudo bibliográfico mais profundo sobre as regras de sequenciamento e realizar uma comparação entre outras regras de sequenciamento para justificar a escolha do modelo utilizado em relação aos resultados. Outra atividade do planejamento e controle da produção que pode ser desenvolvida a partir do presente estudo se trata da elaboração de uma proposta de programação das ordens de produção, bem como definir lotes mínimos de produção, visto que atualmente durante a abertura de ordens, informações como tempo de setup não são levadas em consideração, mesmo que esses fatores influenciem diretamente os custos e rendimento do processo produtivo.

Diante das questões abordadas durante a realização da pesquisa e dos resultados obtidos, foi possível verificar que o estudo poderá colaborar para empresas que possuem recursos limitados no que diz respeito ao planejamento da 
produção, haja vista que essa realidade não é amplamente abordada em pesquisas, o que dificulta o alinhamento da organização em relação a esses conceitos. Apesar dos obstáculos encontrados a relevância do estudo não é reduzida, ao contrário, eles são considerados como meios de aprimoramento dos conhecimentos apresentados até então. Também pode-se notar que para alcançar resultados mais consistentes é necessário considerar todas as variáveis envolvidas no processo, como a organização dos ambientes e a análise do fluxo produtivo.

\section{REFERÊNCIAS}

CONFORTO, E. C.; AMARAL, D. C.; SILVA, S. L. Roteiro para revisão bibliográfica sistemática: aplicação no desenvolvimento de produtos e gerenciamento de projetos. CONGRESSO BRASILEIRO DE GESTÃO DE DESENVOLVIMENTO DE PRODUTO, 8., 2011. [Anais...]. Porto Alegre, 2011.

CHUNG-HSING, Y. A customer-focused planning approach to make-to-order production., Industrial Management \& Data Systems, v. 100, p. 180 - 187, 2000. Disponível em: https://www.emerald.com/insight/content/doi/10.1108/02635570010328693/full/html. Acesso em: 28 ago. 2019. https://doi.org/10.1108/02635570010328693

FATTAHI, P.; MEHRABAD, M. S.; JOLAI, F. Mathematical modeling and heuristic approaches to flexible job shop scheduling problems. Journal of Intelligent Manufacturing, v. 18, n. 3, p. 331- 342, 2007. Disponível em:

https://link.springer.com/article/10.1007/s10845-007-0026-8. Acesso em: 29 ago. 2019. https://doi.org/10.1007/s10845-007-0026-8

FERNANDES, R. O. P. Estudo de sequenciamento da produção em uma indústria de meias. 2006. Disponível em:

http://www.ufff.br/engenhariadeproducao/files/2014/09/2005 3 Rafael.pdf. Acesso em: 16 set. 2019.

GAITHER, Norman; FRAZIER, Greg. Administração da produção e operações. 8. ed. São Paulo: Pioneira Thomson Learning, 2002.

GIL, A. C;. Como elaborar projetos de pesquisa. 4. ed. São Paulo: Atlas, 2002. 176 p.

GOLDRATT, E. M.; FOX, R. E. A corrida pela vantagem competitiva. São Paulo: Pioneira, 1989.

LAKATOS, E. M.; MARCONI, M. A. Metodologia do trabalho científico. 4.ed. São Paulo: Atlas, 1992. $212 \mathrm{p}$.

LUSTOSA, L.; MESQUITA, M. A.; QUELHAS, O.; OLIVEIRA, R. Planejamento e controle da produção. Rio de Janeiro: Elsevier, 2008. 357 p.

MIGUEL, P. A. C.; FLEURY, A.; MELLO, C. H. P.; NAKANO, D. N.; LIMA, E. P.; TURRIONI, J. B.; HO, L. L.; MORABITO, R.; MARTINS, R. A.; SOUSA, R.; COSTA, S. E. G.; PUREZA, 
V. Metodologia de pesquisa em engenharia de produção e gestão de operações. 3. ed. Rio de Janeiro: Elsevier, 2018. 265 p.

MOREIRA, D. A. Administração da produção e operações. 2. ed. rev. São Paulo: Cengage Learning, 2011. $624 \mathrm{p}$.

NOVAS, J. M. Production scheduling and lot streaming at flexible job-shops environments using constraint programming. Computers \& Industrial Engineering , [s. I.], v. 136, p. 252264, 2019. Disponível em:

https://www.sciencedirect.com/science/article/abs/pii/S0360835219304036?via\%3Dihub\#!. Acesso em: 7 out. 2019. https://doi.org/10.1016/i.cie.2019.07.011

PHANDEN, R. K.; JAIN, A.; VERMA, R. A genetic algorithm-based approach for job shop scheduling. Journal of Manufacturing Technology, v. 23, p. 937-946, 2012. Disponível em: https://www.emerald.com/insight/content/doi/10.1108/17410381211267745/full/html. Acesso em: 12 set. 2019. https://doi.org/10.1108/17410381211267745

PARANHOS, R.; FILHO, D. B. F.; ROCHA, E. C.; JÚNIOR, J. A. S.; FREITAS, D. Uma introdução aos métodos mistos. Sociologias, Porto Alegre, p. 384 - 411, 2016. Disponível em: http://www.scielo.br/pdf/soc/v18n42/1517-4522-soc-18-42-00384.pdf. Acesso em: 20 set. 2019. https://doi.org/10.1590/15174522-018004221

PINEDO, M. L. Scheduling: theory, algorithms, ans systems. 3. ed. New York: Springer, 2008. $696 \mathrm{p}$.

PIRES, S. R. I. Gestão da cadeia de suprimentos (Supply Chain Management): conceitos, estratégias, práticas e casos. São Paulo: Atlas, 2004.

ROSS, P.; HART, E.; CORNE, D. Evolutionary scheduling: a review. Genetic Programming and Evolvable Machines, v. 6, n. 2, p. 191-220, 2005. https://doi.org/10.1007/s10710-005$\underline{7580-7}$

RUSSOMANO, V. H. Planejamento e controle da produção. 6. ed. rev. São Paulo: Pioneira, 2000.

SLACK, N.; CHAMBERS, S.; JOHNSTON, R. Administração da produção. 3. ed. rev. São Paulo: Atlas S. A., 2009. 703 p.

SILVA, E. L.; MENEZES, E. M. Metodologia da pesquisa e elaboração de dissertação. 4. ed. Florianópolis: Universidade Federal de Santa Catarina/ UFSC, 2015. 139 p.

TUBINO, D. F. Planejamento e controle da produção: Teoria e prática. 2. ed. São Paulo: Atlas, 2009. 190 p.

ZANELLA, L. C. H. Metodologia de pesquisa. 2. ed. Florianópolis: Departamento de Ciências da Administração/UFSC, 2013. 134 p.

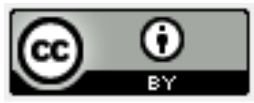

Artigo recebido 28/11/2019 e aceito para publicação em: 07/06/2020 DOI: http://dx.doi.org/10.14488/1676-1901.v20i2.3863 